PROCEEDINGS OF THE

AMERICAN MATHEMATICAL SOCIETY

Volume 126, Number 12, December 1998, Pages 3625-3631

S 0002-9939(98)04615-2

\title{
A NOTE ON TOEPLITZ OPERATORS ON DISCRETE GROUPS
}

\author{
QINGXIANG XU AND XIAOMAN CHEN
}

(Communicated by Palle E. T. Jorgensen)

\begin{abstract}
We study Toeplitz algebras associated to partially-ordered and quasi-partially ordered discrete groups.
\end{abstract}

\section{INTRODUCTION}

In the past thirty years, the classical Toeplitz operators have been extended to various contexts. One important extension is to replace $\left(Z, Z_{+}\right)$by some general ordered (or even just partially ordered) discrete abelian group $\left(G, G_{+}\right)$and study the corresponding Toeplitz operators; for related materials, see [1]-[5]. In recent years, there has been much progress in studying Toeplitz operators on ordered groups. By comparison, less is known about Toeplitz operators on general partially ordered groups. To analyze such Toeplitz operators, a universal Toeplitz algebra is constructed in [1], and this plays a key role in the subsequent work of G. Murphy. It is proved in [1] that when $\left(G, G_{+}\right)$is an ordered group, the universal Toeplitz algebra is isomorphic to the Toeplitz algebra defined in the usual way. However, it is shown in [7] that the analogous statement is not true for Toeplitz algebras on general partially ordered groups.

To study Toeplitz algebras on general partially ordered groups, one approach is the following: given a partially ordered group, find suitable ordered groups that contain the partially ordered group, and then use the Toeplitz algebras on the ordered groups to understand the Toeplitz algebra on the partially ordered group. This approach was first taken by E. Park in [5], and was generalized by the authors in [7] and [8]. To do this, it is necessary to extend partially ordered groups to a more general setting; in [7], quasi-ordered groups are introduced. In this paper, we extend this notion to the nonabelian case in order to study Toeplitz operators on discrete nonabelian groups.

Received by the editors January 10, 1997 and, in revised form, April 24, 1997.

1991 Mathematics Subject Classification. Primary 47B35.

Key words and phrases. Toeplitz operator, discrete group, quasi-partial order.

This research was supported in part by the National Science Foundation of China and the Science and Technology Foundation of Shanghai Higher Education.

(C)1998 American Mathematical Society 


\section{The natural MORPhism Between TOEPLitz Algebras}

Let $G$ be a discrete group, $\left\{\delta_{g} \mid g \in G\right\}$ be the usual orthonormal basis for $\ell^{2}(G)$, where

$$
\delta_{g}(h)= \begin{cases}1, & \text { if } g=h, \\ 0, & \text { otherwise }\end{cases}
$$

for $g, h \in G$. For any $g \in G$, we denote by $L_{g}$ and $R_{g}$ the unitary operators on $\ell^{2}(G)$ defined by

$$
\begin{gathered}
L_{g}\left(\delta_{h}\right)=\delta_{g h}, \\
R_{g}\left(\delta_{h}\right)=\delta_{h g^{-1}}
\end{gathered}
$$

for all $h \in G$.

The von Neumann algebra generated by $\left\{L_{g} \mid g \in G\right\}$ is called the group von Neumann algebra of $G$ and is denoted by $W^{*}(G)$. The $C^{*}$-algebra generated by $\left\{L_{g} \mid g \in G\right\}$ is called the reduced $C^{*}$-algebra of $G$ and is denoted by $C_{r}^{*}(G)$. It is well-known that $W^{*}(G)$ is the commutant of the set $\left\{R_{g} \mid g \in G\right\}$.

For any $E \subseteq G$, let $\ell^{2}(E)$ be the closed subspace of $\ell^{2}(G)$ generated by $\left\{\delta_{g} \mid g \in\right.$ $E\}$; its projection is denoted by $p^{E}$. For any $T \in W^{*}(G)$, we define the compression $T^{(E)}$ of $T$ to $\ell^{2}(E)$ by letting

$$
T^{(E)}=p^{E} T \mid \ell^{2}(E) .
$$

We refer to $T^{(E)}$ as a Toeplitz operator, and call $T$ the symbol of $T^{(E)}$. We also define generalised "unilateral shifts" $T_{g}, g \in G$, by letting

$$
T_{g}=p^{E} L_{g} \mid \ell^{2}(E) \quad(g \in G) .
$$

Definition. The $C^{*}$-algebra generated by $\left\{p^{E} L_{g} p^{E} \mid g \in E\right\}$ is denoted by $\mathcal{T}^{E}(G)$, and is called the Toeplitz algebra with respect to $E$. Its commutator ideal is denoted by $C\left(\mathcal{T}^{E}(G)\right)$.

Let $G_{+} \subseteq G$, we say that $\left(G, G_{+}\right)$is a quasi-partially ordered group (below we briefly denote it by qpo), if $e \in G_{+}, G_{+} \cdot G_{+} \subseteq G_{+}$, and $G=G_{+} \cdot G_{+}^{-1}$, where $e$ is the unit of $G$ and $G_{+}^{-1}=\left\{g^{-1} \mid g \in G_{+}\right\}$. Furthermore if $G=G_{+} \cup G_{+}^{-1}$, then $\left(G, G_{+}\right)$ is referred as a quasi-ordered group. Note that when $G_{+}^{0}=G_{+} \cap G_{+}^{-1}=\{e\}$, then qpo groups are just the usual partially ordered groups.

Natural question. Suppose that $\left(G, G_{1}\right)$ and $\left(G, G_{2}\right)$ are two qpo groups, can the morphism $\gamma^{G_{2}, G_{1}}$ defined by $\gamma^{G_{2}, G_{1}}\left(p^{G_{1}} L_{g} p^{G_{1}}\right)=p^{G_{2}} L_{g} p^{G_{2}}$ for any $g \in G$ be extended as a $C^{*}$-algebra morphism from $\mathcal{T}^{G_{1}}(G)$ to $\mathcal{T}^{G_{2}}(G)$ ?

Note that if it is true, then for any $g_{1} \in G_{1}$,

$$
\begin{gathered}
\gamma^{G_{2}, G_{1}}\left(p^{G_{1}} L_{g_{1}^{-1}} p^{G_{1}} L_{g_{1}} p^{G_{1}}\right)=\gamma^{G_{2}, G_{1}}(1)=\gamma^{G_{2}, G_{1}}\left(p^{G_{1}} L_{e} p^{G_{1}}\right) \\
\text { so } p^{G_{2}} L_{g_{1}^{-1}} p^{G_{2}} L_{g_{1}} p^{G_{2}}=p^{G_{2}} L_{e} p^{G_{2}}=1
\end{gathered}
$$

In particular, $\left(p^{G_{2}} L_{g_{1}^{-1}} p^{G_{2}} L_{g_{1}} p^{G_{2}}\right) \delta_{e}=\delta_{e}$, so $g_{1} \in G_{2}$. Since $g_{1}$ is arbitrary, we know that $G_{1} \subseteq G_{2}$. So in the following we always assume $G_{1} \subseteq G_{2}$.

Definition. Let $E_{1}$ and $E_{2}$ be two subsets of $G$ with $E_{1} \subseteq E_{2}$, we say that $E_{2}$ is finitely decomposed by $E_{1}$ if for any finite subset $F$ of $G$, there exists an element $g \in G$, such that for all $s \in F$, the following hold: 
(a) $s g \in E_{2} \quad$ iff $\quad s \in E_{2}$.

(b) $s g \in E_{1} \cup E_{2}^{C}$.

Theorem 1.1. Let $E_{1}$ and $E_{2}$ be two subsets of $G$ with $E_{1} \subseteq E_{2}$, if $E_{2}$ is finitely decomposed by $E_{1}$, then $\gamma^{E_{2}, E_{1}}$ is well-defined and can be extended as a $C^{*}$-algebra morphism from $\mathcal{T}^{E_{1}}(G)$ to $\mathcal{T}^{E_{2}}(G)$.

Proof. Let $T$ be an operator in $\mathcal{T}^{E_{1}}(G)$ of the form

$$
T=\sum_{i=1}^{m} \xi_{i} \prod_{j=1}^{n_{i}} p^{E_{1}} L_{g_{i j}} p^{E_{1}} ;
$$

then

$$
\gamma^{E_{2}, E_{1}}(T)=\sum_{i=1}^{m} \xi_{i} \prod_{j=1}^{n_{i}} p^{E_{2}} L_{g_{i j}} p^{E_{2}} \quad \text { for } \xi_{i} \in C, g_{i j} \in G .
$$

To show that $\gamma^{E_{2}, E_{1}}$ is well-defined and can be extended as a $C^{*}$-algebra morphism, it suffices to show that $\left\|\gamma^{E_{2}, E_{1}}(T)\right\| \leq\|T\|$, since such kind of $T$ is dense in $\mathcal{T}^{E_{1}}(G)$.

$\forall \varepsilon>0$, there exists some $f \in \ell^{2}\left(E_{2}\right)$ with finite support such that $\|f\|=1$, and $\left\|\gamma^{E_{2}, E_{1}}(T)\right\| \leq\left\|\gamma^{E_{2}, E_{1}}(T) f\right\|+\varepsilon$.

Let $f=\sum_{p=1}^{n} \eta_{p} \delta_{h_{p}}$, set $F=\left\{h_{p},\left(\prod_{j=m_{i}}^{n_{i}} g_{i j}\right) h_{p} \mid p=1,2, \ldots, n, i=1,2, \ldots, m\right.$, $\left.1 \leq m_{i} \leq n_{i}\right\}$. By assumption, there exists an element $g \in G$, such that for all $s \in F$, we have

$$
\begin{gathered}
s g \in E_{2} \quad \text { iff } \quad s \in E_{2}, \\
s g \in E_{1} \cup E_{2}^{C} .
\end{gathered}
$$

By (1.1), we know that

$$
R_{g^{-1}} \gamma^{E_{2}, E_{1}}(T) f=\gamma^{E_{2}, E_{1}}(T) R_{g^{-1}} f .
$$

By (1.2), we have

$$
\begin{aligned}
& \gamma^{E_{2}, E_{1}}(T) R_{g^{-1}} f=\left(\sum_{i=1}^{m} \xi_{i} \prod_{j=1}^{n_{i}} p^{E_{2}} L_{g_{i j}} p^{E_{2}}\right) R_{g^{-1}} f \\
& =\left(\sum_{i=1}^{m} \xi_{i} \prod_{j=1}^{n_{i}} p^{E_{2}} p^{E_{1} \cup E_{2}^{C}} L_{g_{i j}} p^{E_{2}} p^{E_{1} \cup E_{2}^{C}}\right) R_{g^{-1}} f=T R_{g^{-1}} f
\end{aligned}
$$

so

$$
\begin{aligned}
\left\|\gamma^{E_{2}, E_{1}}(T)\right\| & \leq\left\|\gamma^{E_{2}, E_{1}}(T) f\right\|+\varepsilon \\
& =\left\|R_{g^{-1}} \gamma^{E_{2}, E_{1}}(T) f\right\|+\varepsilon=\left\|T R_{g^{-1}} f\right\|+\varepsilon \leq\|T\|+\varepsilon .
\end{aligned}
$$

Remark. When $E_{2}$ is finitely decomposed by $E_{1}$ and $e \in E_{2}$, then for any finite subset $F$ of $G$, we can further choose $g \in E_{1}$ satisfying the condition. In fact, replacing $F$ by $F \cup\{e\}$ will do. So for qpo groups $\left(G, G_{1}\right)$ and $\left(G, G_{2}\right)$ with $G_{1} \subseteq G_{2}, G_{2}$ is finitely decomposed by $G_{1}$, iff for any finite subset $F=F_{1} \cup F_{2}$ with $F_{1} \subseteq G_{2} \backslash G_{1}$ and $F_{2} \subseteq G_{2}^{C}$, there exists $g \in G_{1}$ such that $F_{1} g \subseteq G_{1}$ and $F_{2} g \subseteq G_{2}^{C}$. 
Definition. For any subset $E$ of $G$, we say $E$ is finitely liftable, if $G$ is finitely decomposed by $E$.

Examples. (1) Let $\left(G, G_{+}\right)$be a qpo group, then $G_{+}$is finitely liftable. In fact, it is easy to show inductively that for any finite subset $F$ of $G$, there is a $g$ in $G_{+}$ such that $F g \subseteq G_{+}$.

(2) Recall for any subset $S$ of $G, S$ is said to be almost invariant, if $S \backslash g S$ is finite for all $g \in G$. By Lemma 4.1 in [6], we know that if $S$ is almost invariant, then $S$ is finitely liftable.

Proposition 1.2. For any subset $E$ of $G$, if $E$ is finitely liftable, then we have the following exact sequence:

$$
0 \longrightarrow \operatorname{Ker}^{G, E} \longrightarrow \mathcal{T}^{E}(G) \stackrel{\gamma^{G, E}}{\longrightarrow} C_{r}^{*}(G) \longrightarrow 0
$$

with the property that

(1) $\operatorname{Ker} \gamma^{G, E} \subseteq K\left(\ell^{2}(E)\right)$ if and only if $E$ is almost invariant, where $K\left(\ell^{2}(E)\right)$ is the ideal of compact operators on $\ell^{2}(E)$.

(2) If $(G, E)$ is a pqo group, then $\operatorname{Ker} \gamma^{G, E} \subseteq C\left(\mathcal{T}^{E}(G)\right)$ and $\operatorname{Ker} \gamma^{G, E}=$ $C\left(\mathcal{T}^{E}(G)\right)$ if and only if $G$ is abelian. In this case, we have the following exact sequence:

$$
0 \longrightarrow C\left(\mathcal{T}^{E}(G)\right) \longrightarrow \mathcal{T}^{E}(G) \stackrel{\sigma^{E}}{\longrightarrow} C(\widehat{G}) \longrightarrow 0
$$

where $\widehat{G}$ is the Pontryagin dual group of $G, \sigma^{E}\left(p^{E} L_{g} p^{E}\right)=\varepsilon_{g}$ for $g \in G$, and $\varepsilon_{g}(\gamma)=\gamma(g)$ for $\gamma \in \widehat{G}$.

Proof. By Theorem 1.1 we know that $\gamma^{G, E}$ is well-defined and can be extended as a $C^{*}$-algebra morphism, so we have the following exact sequence:

$$
0 \longrightarrow \operatorname{Ker} \gamma^{G, E} \longrightarrow \mathcal{T}^{E}(G) \stackrel{\gamma^{G, E}}{\longrightarrow} C_{r}^{*}(G) \longrightarrow 0 .
$$

Define a bounded linear operator $\rho^{E}$ from $C_{r}^{*}(G)$ to $B\left(\ell^{2}(E)\right)$ by $\rho^{E}(T)=p^{E} T p^{E}$ for $T \in C_{r}^{*}(G)$, then the $C^{*}$-algebra generated by $\rho^{E}\left(C_{r}^{*}(G)\right)$ equals $\mathcal{T}^{E}(G)$ and $\gamma^{G, E} \circ \rho^{E}=1$, so $\operatorname{Ker} \gamma^{G, E}=\left\{T-\rho^{E} \circ \gamma^{G, E}(T) \mid T \in \mathcal{T}^{E}(G)\right\}$.

(1) Since $\operatorname{Ker} \gamma^{G, E}=\left\{T-\rho^{E} \circ \gamma^{G, E}(T) \mid T \in \mathcal{T}^{E}(G)\right\}$, it easily follows that $\operatorname{Ker} \gamma^{G, E} \subseteq K\left(\ell^{2}(E)\right)$ if and only if $E$ is almost invariant.

(2) If $(G, E)$ is a qpo group, let $T=\prod_{i=1}^{n} p^{E} L_{g_{i}} p^{E}, g_{i} \in G$ for $i=1,2, \ldots, n$, then $T-\rho^{E} \circ \gamma^{G, E}(T)=\prod_{i=1}^{n} p^{E} L_{g_{i}} p^{E}-p^{E} L_{g_{1} g_{2} \ldots g_{n}} p^{E}$. Let $F=\left\{g_{n}, g_{n-1} g_{n}, \ldots\right.$, $\left.g_{1} g_{2} \ldots g_{n}\right\}$, since $E$ is finitely liftable, there is a $g$ in $E$, such that $F g \subseteq E$. Let $S=1-p^{E} L_{g} p^{E} L_{g^{-1}} p^{E}$, then $S \in C\left(\mathcal{T}^{E}(G)\right)$ and it is easy to show that $\left(T-\rho^{E} \circ \gamma^{G, E}(T)\right) S=T-\rho^{E} \circ \gamma^{G, E}(T)$, which implies that $T-\rho^{E} \circ \gamma^{G, E}(T)$ belongs to $C\left(\mathcal{T}^{E}(G)\right)$. Since the linear span of such kind of $T$ is dense in $\mathcal{T}^{E}(G)$, by the continuity of $\rho^{E}$ and $\gamma^{G, E}$, we know that $\operatorname{Ker} \gamma^{G, E} \subseteq C\left(\mathcal{T}^{E}(G)\right)$. If $\operatorname{Ker} \gamma^{G, E}=$ $C\left(\mathcal{T}^{E}(G)\right)$, then for any $g_{1}, g_{2} \in G, T=p^{E} L_{g_{1}} p^{E} L_{g_{2}} p^{E}-p^{E} L_{g_{2}} p^{E} L_{g_{1}} p^{E} \in$ $C\left(\mathcal{T}^{E}(G)\right)$, so $\gamma^{G, E}(T)=0$, which implies $g_{1} g_{2}=g_{2} g_{1}$, so $G$ is abelian. On the otherhand, if $G$ is abelian, then $C_{r}^{*}(G)$ is abelian, and since $\mathcal{T}^{E}(G) / \operatorname{Ker} \gamma^{G, E}$ is isomorphic to an abelian $C^{*}$-algebra, we know that in this case $C\left(\mathcal{T}^{E}(G)\right) \subseteq \operatorname{Ker} \gamma^{G, E}$, so $\operatorname{Ker} \gamma^{G, E}=C\left(\mathcal{T}^{E}(G)\right)$.

When $G$ is abelian, it is amenable which implies that the regular representation of $C^{*}(G) \cong C(\widehat{G})(\widehat{G}$ is compact since $G$ is discrete and abelian) is faithful. For any $g \in G$, the regular representation of $\delta_{g}$ is $L_{g}$, and its Fourier transform is $\varepsilon_{g}$, thus the conclusion holds. 
Next we show that the condition of finitely decomposable stated in Theorem 1.1 in some sense is also necessary.

Let $\left(G, G_{1}\right)$ and $\left(G, G_{2}\right)$ be two qpo groups with $G_{1} \subseteq G_{2}$, define a linear operator $\rho: \mathcal{T}^{G_{2}}(G) \rightarrow B\left(\ell^{2}\left(G_{1}\right)\right)$ by $\rho(T)=p^{G_{1}} T p^{G_{1}}, T \in \mathcal{T}^{G_{2}}(G)$. The $C^{*}$-subalgebra of $B\left(\ell^{2}\left(G_{1}\right)\right)$ generated by $\rho\left(\mathcal{T}^{G_{2}}(G)\right)$ is denoted by $\mathcal{R}^{G_{1}}(G)$. Clearly $\mathcal{R}^{G_{1}}(G)$ contains $\mathcal{T}^{G_{1}}(G)$ and a dense subalgebra of $\mathcal{R}^{G_{1}}(G)$ consists of operators of the form

$$
T=\sum_{l=1}^{N} \xi_{l}\left(\prod_{i=1}^{n_{l}} p^{G_{1}}\left(\prod_{j=1}^{k_{i}} p_{l i j} L_{g_{l i j}}\right) p^{G_{1}}\right)
$$

where each $p_{l i j}$ equals $p^{G_{1}}$ or $p^{G_{2}}$.

Proposition 1.3. Let $\left(G, G_{1}\right)$ and $\left(G, G_{2}\right)$ be two qpo groups with $G_{1} \subseteq G_{2}$, then the natural morphism $\gamma$ defined by $\gamma(T)=\sum_{l=1}^{N} \xi_{l}\left(\prod_{i=1}^{n_{l}} p^{G_{2}}\left(\prod_{j=1}^{k_{i}} p^{G_{2}} L_{g_{l i j}}\right) p^{G_{2}}\right)$ can be extended as a $C^{*}$-algebra morphism from $\mathcal{R}^{G_{1}}(G)$ to $\mathcal{T}^{G_{2}}(G)$, if and only if $G_{2}$ is finitely decomposed by $G_{1}$.

Proof. If $\gamma$ is well-defined and can be extended as a $C^{*}$-algebra morphism, we show that in this case $G_{2}$ is finitely decomposed by $G_{1}$. If not, then there exists a finite subset $F=F_{1} \cup F_{2}$ with $F_{1} \subseteq G_{2} \backslash G_{1}$ and $F_{2} \subseteq G_{2}^{C}$, such that for any $g \in G_{1}$, either $\left(F_{1} g\right) \cap G_{1}^{C} \neq \emptyset$ or $\left(F_{2} g\right) \cap G_{2} \neq \emptyset$. Since $e \in G_{1}, G_{1} \cdot G_{1} \subseteq G_{1}$, and $G=G_{1} \cdot G_{1}^{-1}$, it is easy to show that both $F_{1}$ and $F_{2}$ are non-empty. Let $F_{1}=\left\{g_{1}, g_{2}, \ldots, g_{n}\right\}$ and $F_{2}=\left\{h_{1}, h_{2}, \ldots, h_{l}\right\}$.

For any $g \in G$, define $T_{g}^{(i)}=p^{G_{i}} L_{g^{-1}} p^{G_{i}} L_{g} p^{G_{i}}$ for $i=1,2$; set

$$
T=T_{g_{1}}^{(1)} T_{g_{2}}^{(1)} \ldots T_{g_{n}}^{(1)}\left(1-T_{h_{1}}^{(2)}\right)\left(1-T_{h_{2}}^{(2)}\right) \ldots\left(1-T_{h_{l}}^{(2)}\right) p^{G_{1}} \in \mathcal{R}^{G_{1}}(G) .
$$

Then it is easy to show that $T=0$, so $\gamma(T)=0$.

On the other hand, $\gamma(T)=T_{g_{1}}^{(2)} T_{g_{2}}^{(2)} \ldots T_{g_{n}}^{(2)}\left(1-T_{h_{1}}^{(2)}\right)\left(1-T_{h_{2}}^{(2)}\right) \ldots\left(1-T_{h_{l}}^{(2)}\right) p^{G_{2}}$ and $\gamma(T) \delta_{e}=\delta_{e} \neq 0$, which is a contradiction.

For the sufficient part, see the proof of Theorem 1.1.

Proposition 1.4. Let $\left(G, G_{1}\right)$ and $\left(G, G_{2}\right)$ be two qpo groups with $G_{1} \subseteq G_{2}$, if $\left(G, G_{1}\right)$ is quasi-ordered, then $G_{2}$ is finitely decomposed by $G_{1}$.

Proof. For any finite subset $F$ of $G$, let $F=\left\{g_{1}, g_{2}, \ldots, g_{n}, h_{1}, h_{2}, \ldots, h_{l}\right\}$ with $\left\{g_{1}, g_{2}, \ldots, g_{n}\right\} \subseteq G_{2}$ and $\left\{h_{1}, h_{2}, \ldots, h_{l}\right\} \subseteq G_{2}^{C}$. Let $g_{1}, g_{2}, \ldots, g_{k} \in G_{1}$, and $g_{k+1}, g_{k+2}, \ldots, g_{n} \in G_{2} \backslash G_{1}$, then $g_{k+1}, g_{k+2}, \ldots, g_{n} \in G_{2} \cap G_{1}^{-1} \subseteq G_{2}^{0}$. Since $G_{2}^{C} \cdot G_{2}^{0}=G_{2}^{C}$, it remains only to prove that for any finite subset $\left\{l_{1}, l_{2}, \ldots, l_{m}\right\} \subseteq$ $G_{2} \cap G_{1}^{-1}$, there is a $g \in G_{2}^{0} \cap G_{1}$ such that $l_{i} g \in G_{1}$ for all $i$. When $m=1, l_{1} \in$ $G_{2} \cap G_{1}^{-1}$, set $g=l_{1}^{-1}$ will do. If for $\left\{l_{1}, l_{2}, \ldots, l_{m-1}\right\}$, there is a $g^{\prime} \in G_{2}^{0} \cap G_{1}$, such that $l_{1} g^{\prime}, l_{2} g^{\prime}, \ldots, l_{m-1} g^{\prime} \in G_{1}$, in this case, if $l_{m} g^{\prime} \in G_{1}$, then set $g=g^{\prime}$; otherwise $l_{m} g^{\prime} \in G_{1}^{-1} \cap G_{2}^{0}$, so $\left(l_{m} g^{\prime}\right)^{-1} \in G_{1} \cap G_{2}^{0}$. Let $g=g^{\prime}\left(l_{m} g^{\prime}\right)^{-1}$, then $l_{i} g \in G_{1}$ for all $i$.

Proposition 1.5. If $G \neq\{e\}$, let $\left(G, G_{1}\right)$ and $\left(G, G_{2}\right)$ be two ordered groups, define $G_{+}^{2}=G_{1} \times G_{2}, G_{\ell e x}^{2}=\left\{\left(g_{1}, g_{2}\right) \mid g_{1} \in G_{1} \backslash\{e\}\right.$ or $g_{1}=e$ and $\left.g_{2} \in G_{2}\right\}$, then $\gamma^{G_{\ell e x}^{2}, G_{+}^{2}}$ cannot be extended as a $C^{*}$-algebra morphism. 
Proof. Let $E_{1}=G_{1} \times G$ and $E_{2}=G \times G_{2}$, then $E_{1} \cap E_{2}=G_{+}^{2}$. If $\gamma^{G_{\ell e x}^{2}}, G_{+}^{2}$ is well-defined and can be extended, then choose $g_{1} \in G_{1} \backslash\{e\}$, and $g_{2} \in G_{2} \backslash\{e\}$, let $T=p^{E_{1}} p^{E_{2}} L_{\left(g_{1}, g_{2}\right)} p^{E_{2}} L_{\left(g_{1}^{-1}, g_{2}^{-1}\right)} p^{E_{1}} p^{E_{2}}$, we have

$$
\begin{aligned}
T & =p^{E_{1}} p^{E_{2}} L_{\left(g_{1}, g_{2}\right)} L_{\left(g_{1}^{-1}, e\right)} p^{E_{2}} L_{\left(g_{1}, e\right)} L_{\left(g_{1}^{-1}, g_{2}^{-1}\right)} p^{E_{1}} p^{E_{2}} \\
& =p^{E_{1}} p^{E_{2}} L_{\left(e, g_{2}\right)} p^{E_{2}} L_{\left(e, g_{2}^{-1}\right)} p^{E_{1}} p^{E_{2}} \\
& =p^{E_{1}} p^{E_{2}} L_{\left(e, g_{2}\right)} p^{E_{1}} p^{E_{2}} L_{\left(e, g_{2}^{-1}\right)} p^{E_{1}} p^{E_{2}},
\end{aligned}
$$

so $T \in \mathcal{T}^{G_{+}^{2}}\left(G^{2}\right)$, and

$$
\gamma^{G_{\ell e x}^{2}, G_{+}^{2}}(T)=p^{G_{\ell e x}^{2}} L_{\left(e, g_{2}\right)} p^{G_{\ell e x}^{2}} L_{\left(e, g_{2}^{-1}\right)} p^{G_{\ell e x}^{2}} .
$$

On the other hand,

$$
\begin{gathered}
T=p^{E_{1}} p^{E_{2}} L_{\left(g_{1}, g_{2}\right)} L_{\left(g_{1}^{-2}, e\right)} p^{E_{2}} L_{\left(g_{1}^{2}, e\right)} L_{\left(g_{1}^{-1}, g_{2}^{-1}\right)} p^{E_{1}} p^{E_{2}} \\
=p^{G_{+}^{2}} L_{\left(g_{1}^{-1}, g_{2}\right)} p^{G_{+}^{2}} L_{\left(g_{1}, g_{2}^{-1}\right)} p^{G_{+}^{2}},
\end{gathered}
$$

thus we have

$$
p^{G_{\ell e x}^{2}} L_{\left(g_{1}^{-1}, g_{2}\right)} p^{G_{\ell e x}^{2}} L_{\left(g_{1}, g_{2}^{-1}\right)} p^{G_{\ell e x}^{2}}=1 .
$$

So $\gamma^{G_{\ell e x}^{2}, G_{+}^{2}}(T)=1$, which is a contradiction since $\left(e, g_{2}^{-1}\right) \notin G_{\ell e x}^{2}$.

\section{TOEPLITZ OPERATORS ON DIRECTED SETS}

Let $E$ be a subset of $G$, in this section, we always assume the following two conditions hold:

(1) $E$ is a directed set, the partial order on it is denoted by $\leq$.

(2) For any finite subset $F$ of $G$, there is a $g_{0} \in E$ such that $F g \subseteq E$ for all $g \geq g_{0}$.

Examples. (1) Let $(G, E)$ be a partially ordered group, for any $g_{1}, g_{2} \in G$, we say that $g_{1} \leq g_{2}$ if $g_{1}^{-1} g_{2} \in E$. Since for any $g_{1}, g_{2} \in E$, there is a $g \in E$ such that $g_{1}^{-1} g \in E$ and $g_{2}^{-1} g \in E, E$ is a directed set and satisfies condition (2) above.

(2) Let $E$ be a countably infinite subset of $G$, if $E$ is almost invariant, then $E$ will satisfy the conditions.

By definition, we know that $E$ is finitely liftable, so we have the following exact sequence:

$$
0 \longrightarrow \operatorname{Ker} \gamma^{G, E} \longrightarrow \mathcal{T}^{E}(G) \longrightarrow C_{r}^{*}(G) \longrightarrow 0 .
$$

So for any $T \in C_{r}^{*}(G)$, we have $\|T\|=\left\|\rho^{E}(T)\right\|=\left\|T^{(E)}\right\|$, it still holds for all $T \in W^{*}(G)$, since it easily shows that $\lim _{g \in E} R_{g} p^{E} R_{g}^{*}=1$ in the strong operator topology on $B\left(\ell^{2}(G)\right)$. Replace $K\left(\ell^{2}(E)\right)$ or $C\left(\mathcal{T}^{E}(G)\right)$ by general $K e r \gamma^{G, E}$, a unified method to deal with Toeplitz operators on directed sets can be given. For details, see [6] and Section 3 of [1]. 


\section{REFERENCES}

1. G. Murphy, Ordered groups and Toeplitz algebras, J. Operator Theory 18 (1987), 303-326. MR 89f: 46132

2. G. Murphy, Spectral and index theory for Toeplitz operators, Proc. Royal Irish Acad., 91 (A) (1991), 1-6. MR 93k:47039

3. G. Murphy, An index theorem for Toeplitz operators, J. Operator Theory 29 (1993), 97-114. MR 95h: 47035

4. G. Murphy, Toeplitz operators and algebras, Math. Z. 208 (1991), 355-362. MR 93e:46072

5. E. Park, Index theory and Toeplitz algebras on certain cones in $Z^{2}$, J. Operator Theory 23 (1990), 125-146. MR 91i:47039

6. M. Pavone, Toeplitz operators on discrete groups, J. Operator Theory 27 (1992), 359-384. MR 94k: 47043

7. X. Chen and Q. Xu, Toeplitz operators on discrete abelian groups, preprint.

8. X. Chen and Q. Xu, Toeplitz operators determined by multiple quasi-partial ordered groups, preprint.

9. Q. Xu, Toeplitz operators on the Bergman space and the Hardy space, Ph.D. Thesis, Fudan, Univ., Shanghai, 1995.

Department of Mathematics, Shanghai Normal University, Shanghai, 200234, PeoPLE'S REPUBLIC OF CHINA

E-mail address: mathsci@dns.shtu.edu.cn

Institute of Mathematics, Fudan University, Shanghai, 200433, People's Republic of CHINA

E-mail address: xchen@fudan.edu.cn 